\title{
Near-Infrared Spectroscopy for Anticounterfeiting Innovative Fibers
}

\author{
Jing Cao and Suraj Sharma \\ Department of Textiles, Merchandising and Interiors, University of Georgia, 350 Dawson Hall, Athens, GA 30602, USA
}

Correspondence should be addressed to Suraj Sharma; ssharma@uga.edu

Received 30 April 2013; Accepted 6 June 2013

Academic Editors: G. Schoukens and C. Wang

Copyright (C) 2013 J. Cao and S. Sharma. This is an open access article distributed under the Creative Commons Attribution License, which permits unrestricted use, distribution, and reproduction in any medium, provided the original work is properly cited.

Near-infrared (NIR) spectroscopy has gained increased attention for the qualitative and quantitative evaluation of textile and polymer products. Many NIR instruments have been commercialized to identify the natural and synthetic fibers; however, there is a strong need to have NIR database of these high-performance fibers to detect contraband textile materials rapidly and quantitatively. In this study, NIR spectra of PLA, Kevlar, Spandex and Sorona woven fabrics were collected and studied by several calibration models to identify the fibers. The results indicated that these four innovative fibers had been successfully distinguished by their NIR spectra in combination with preprocessing of $1 / X$ transformation, SNV, and 2nd Savitzky-Golay derivative as well as principalcomponent-analysis (PCA-) based chemometric methods. Our promising results suggest that NIR spectroscopy is an effective technique to anticounterfeit innovative fibers.

\section{Introduction}

Recently, near-infrared (NIR) spectroscopy has gained increased attention for the qualitative and quantitative evaluation of textile and polymer products. The NIR spectroscopy region extends from approximately $800 \mathrm{~nm}$ to $2500 \mathrm{~nm}$ in the electromagnetic spectrum. Different materials absorb NIR energy at different wavelengths. When radiation is absorbed, NIR spectroscopy measures overtones and combinations of the fundamental molecular vibrational transitions that occur in the mid-infrared region. These absorbance wavelengths (and the corresponding frequencies) form a unique NIR signature depending on the chemical and physical properties of the materials. This method is nondestructive and environmentally friendly. Over the past decades, NIR spectroscopy has become a widely used analytical technique for process control, for quality assessment, and for determining the unknowns of complex mixture [1].

NIR analysis is a simple, rapid, and accurate technique for studying the properties and characteristics of textile materials. Many NIR instruments have been commercialized to identify the natural and synthetic fibers, such as cotton, rayon, nylon, polyester, and poly(vinyl alcohol) [2]. Ghosh and Roy [3] used homologs of cotton to develop a calibration equation for monitoring the sugar content in cotton; Gosh and Rodgers [4] and Tincher et al. [5] investigated the heatset temperature of nylon carpet and its heat history using NIR spectroscopy. By using advanced diagnostic statistics and computer programs, Richard et al. [6] and Jasper and Kovacs [7] demonstrated the qualitative classification of various natural and synthetic fibers to reveal subtle differences among NIR spectra in the set of samples. Sohn et al. [8] and Ruckebusch et al. [9] used NIR spectroscopy in quantitative analyses of linen/cotton and cotton/polyester blends, respectively.

Since the first synthetic fiber, nylon, was invented in 1935, manmade fibers have developed rapidly beyond the traditional applications for apparel and home furnishings. Today, manmade fibers account for 60.7 percent of the world's fiber consumption [10]. In 2005, 13 million tons of textile products, especially those catering to technical performance and functional properties, were primarily utilized in civil engineering, the automotive industry, the aerospace industry, and medical and healthcare products [11]. To satisfy the requirements of these products, a large number of innovative fibers have been invented, exhibiting high end performance in chemical, physical, or mechanical properties. These fibers include Kevlar, Nomex, Spectra fiber, Vectran, Sulfar, PBO 


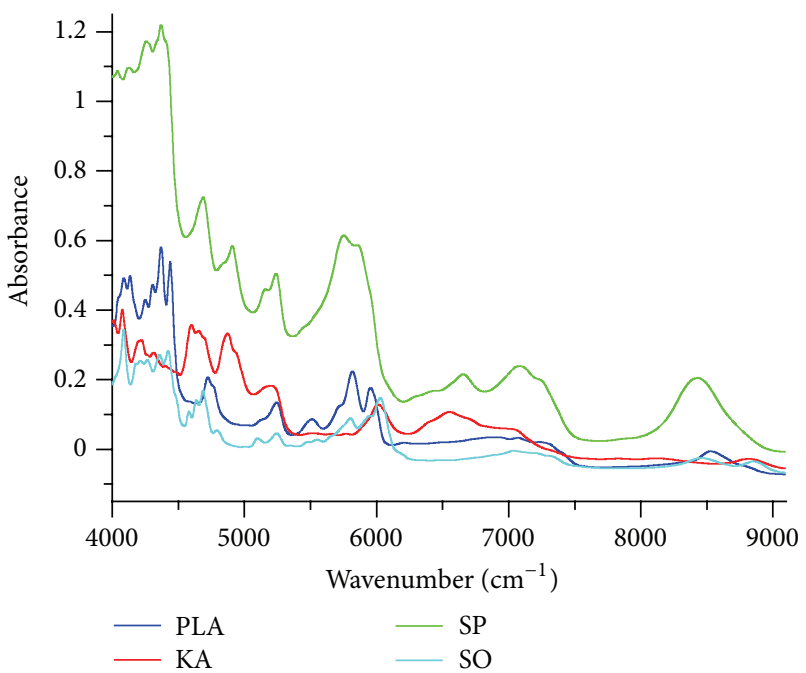

(a)

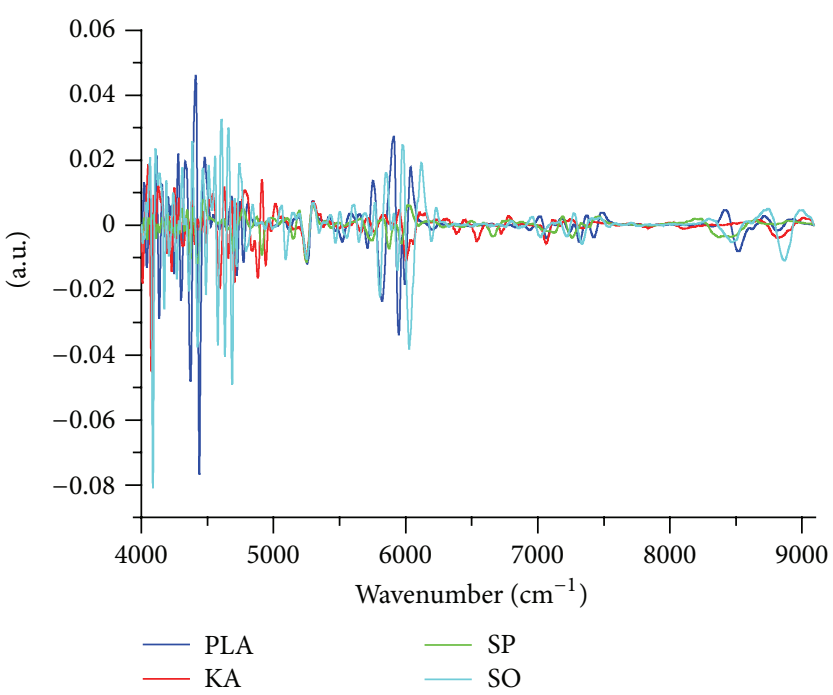

(b)

Figure 1: Near-infrared spectra for PLA, Kevlar (KA), Spandex (SP), and Sorona (SO) fibers from data after (a) $1 / X$ transformation, (b) $1 / X$ transformation, SNV, and 2nd Savitzky-Golay derivative.

fiber, and PLA fiber. Given their high price and advanced end use, anticounterfeiting techniques, such as using NIR spectroscopy, for these innovative fibers is of definite importance. Several high-performance and smart textiles are under development for enhancing human performance, health, and comfort. For this reason, product designers are experimenting with several heterogeneous textile materials, including their blends. Although commercial NIR spectroscopy manufactures for fiber identification are able to update new/modified fibers, these spectra must be precalibrated by the vendor, and there is a concern that new fibers could be successfully added to instrument's identification routines. Therefore, there is a strong need to have an NIR database of these high-performance fiber materials for anticounterfeiting in order to protect consumers from fake tagged products. The long-term research goal is to establish an NIR library of calibrating spectra for high-performance, innovative fibers to detect contraband textile materials rapidly and quantitatively. The objective of this study was to use NIR spectroscopy in combination with chemometric methods to identify fibers. In this study, NIR measurements were conducted on some innovative commercial fibers in order to determine whether NIR spectroscopy can ensure the identification of anticounterfeit fibers tagged as high-performance fibers, after determining their precalibration spectra. The optimal chemometric model for calibration was also investigated. NIR spectra from the testing of some innovative fibers collected via FT-NIR spectroscopy (see facilities and resources) will eventually establish an NIR spectral library that will help in recognizing unknowns from a library spectra of known fibers. To find the best calibration model, spectra data from samples were imported into chemometric analysis software to conduct derivative treatments for reducing baseline interference and increasing the resolution of small absorbance bands.

\section{Experimental}

2.1. Materials. Four innovative fibers have been purchased from major supplied companies: PLA (poly(lactide)), Kevlar (poly(para-phenylene terephthalamide)), Spandex (poly(urethane)), and Sorona (poly(trimethylene terephthalate)) to determine whether near-infrared spectroscopy is an effective technique to differentiate these fibers. All testing samples were made of woven structures and randomly selected from the market irrespective of their physicochemical properties. The samples were conditioned at $21.1^{\circ} \mathrm{C}$ and $65 \%$ relative humidity overnight before spectra measurement.

2.2. Near-Infrared Apparatus and Spectra Collection. NIR spectra of these fibers were recorded in the transmittance mode using an NIR spectroscopy (FOSS NIRSystems, Model 6500). The fiber samples were placed to completely cover the bottom of sample holder in order to measure their NIR spectra. Sample spectra were obtained over the wavelength range $1,100-2,500 \mathrm{~nm}$ (wavenumber range $4000-9100 \mathrm{~cm}^{-1}$ ). The NIR instrument was controlled, and spectra were obtained, by using the software Vision version 2.51. The raw spectra displayed after acquisition are reflectance spectra transformed into absorbance spectra by a logarithmic function $(\log 1 / R)$ based on the Lambert-Beer law.

2.3. Chemometric Analysis. In this study, three preprocessing methods were applied to raw spectral data by using software Unscrambler $X$ (Camo, Norway), which was $1 / X$ transformation, standard variate (SNV), and 2nd SavitzkyGolay derivative. The $1 / X$ transformation transforms the data points from wavelength units into wavenumber units, resulting in a more realistic presentation [12]. SNV was used 


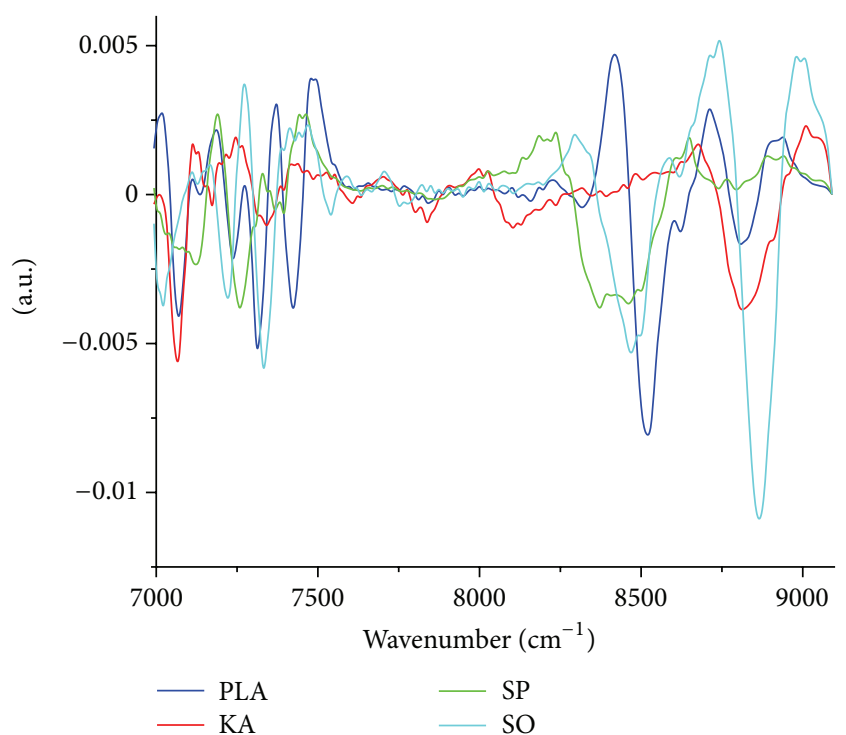

(a)

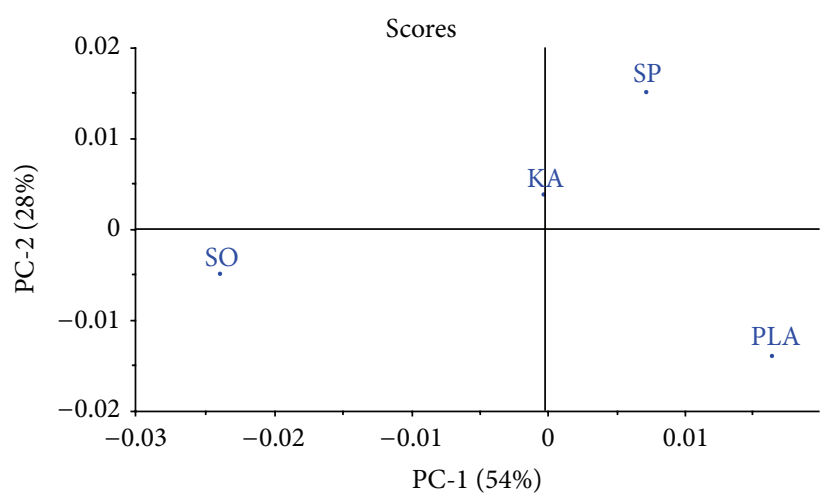

(b)

FIGURE 2: (a) Selected NIR spectra region between 7100 and $9100 \mathrm{~cm}^{-1}$ for PCA analysis and (b) score plot from PCA analysis of PLA, Kevlar (KA), Spandex (SP), and Sorona (SO) fibers after 1/X transformation, SNV, and 2nd Savitzky-Golay derivative.

to remove slope variation and to correct scatter effects in reflectance spectroscopy. 2nd Savitzky-Golay derivative was taken to reduce baseline offset and to enhance the small spectral differences. However, this procedure also amplifies spectral noise and is therefore combined with a SavitzkyGolay algorithm, which includes a smoothing function [13]. Principal component analysis (PCA) was used to indicate the data trend and distinguish useful information from noise and meaningless variation.

\section{Results and Discussion}

3.1. Spectra Investigation. Figure 1(a) shows the NIR spectra of four studied fibers for the data after $1 / X$ transformation. While the curve shows varying shapes, the differentiation among these fiber is not visible. Then, the combination of a serial of preprocessing methods has been determined in order to produce a principal component analysis (PCA) model separating these fibers. Figure 1(b) shows the spectra of each fiber after $1 / X$ transformation, SNV, and 2nd Savitzky-Golay derivative. The wavenumber region from 5155 to $7000 \mathrm{~cm}^{-1}$ was excluded in analysis to avoid the influence of water absorption bands, which has strong overtones in this region [12]. Additionally, some regions exhibiting a high noise level (e.g., $4000-5000 \mathrm{~cm}^{-1}$ and $5800-6200 \mathrm{~cm}^{-1}$ ) should be also excluded, as shown in Figure 1(b). Therefore, the spectral regions between 7100 and $9100 \mathrm{~cm}^{-1}$ were selected for multivariate analysis based on PCA model.

3.2. Principal Components Analysis (PCA). PCA is a simple, nonparametric method for an explorative analysis of spectroscopic data set characterized by highly collinear variables, which enable to reduce a complex data set to a lower dimension to reveal the differentiation [14]. In particular, outliers in data set can be detected from PCA score plots by the Hotelling's $T^{2}$-statistic and by the Q-statistic [15]. Basically, there are two score vectors (PC1 and PC2) calculated by the NIPALS algorithm in PCA analysis based on the difference of multiple variables. To discriminate several materials, score vectors are plotted against each other. If score points for different materials are clearly separated from each other, the identification is possible [16]. Figure 2 shows PCA score plot (PC1 and PC2) of data set for all tested innovative fibers after previous preprocessing methods and wavenumber selection. It appeared that the complete separation of PLA, Kevlar, Spandex, and Sorona fibers were achieved by PCA processing. The first two principal components account for $82 \%$ of the variance, thus describing the main structure of data set. Therefore, the preprocessing methods for sample spectra in this study, which were $1 / X$ transformation, SNV, and 2nd Savitzky-Golay derivative, are effective to ensure the identification of studied fibers by using PCA analysis. However, more samples of each fiber should be studied for validation of this identification model.

\section{Conclusions}

In this study, PLA, Kevlar, Spandex, and Sorona fibers have been successfully distinguished by using NIR spectroscopy after preprocessing of $1 / X$ transformation, SNV, and 2nd Savitzky-Golay derivative and PCA multivariate analysis. Therefore, we suggested that NIR spectroscopy in combination with appropriate chemometric methods could be an effective technique to anticounterfeit innovative fibers. In the future studies, the NIR database of more innovative fibers can 
be compiled, and more spectra of each innovative fiber should be studied for validating of identification model.

\section{References}

[1] E. W. Ciurczak, "Principles of near-infrared spectroscopy", in Handbook of Near-Infrared Analysis, vol. 7, 2001.

[2] J. E. Rodgers, "Influences of carpet and instrumental parameters on the identification of carpet face fiber by NIR," AATCC Review, vol. 2, no. 6, pp. 27-32, 2002.

[3] S. Ghosh and R. B. Roy, "Quantitative near-infra-red analysis of reducing sugar from the surface of cotton," Journal of the Textile Institute, vol. 79, no. 3, pp. 504-510, 1988.

[4] S. Ghosh and J. E. Rodgers, "Determining heatset temperature by near-infrared reflectance spectroscopy," Textile Research Journal, vol. 55, no. 9, pp. 556-560, 1985.

[5] W. C. Tincher, P. G. Casey, and A. R. Mayfield, "Characterization of heat set nylon carpet yarn," Textile Chemist and Colorist, vol. 18, no. 2, pp. 25-30, 1986.

[6] G. W. Richard, E. G. Mark, and L. S. Robert, "Near-infrared spectrum qualification via mahalanobis distance determination," Applied Spectroscopy, vol. 41, no. 7, pp. 1204-1213, 1987.

[7] W. J. Jasper and E. T. Kovacs, "Using neural networks and NIR spectrophotometry to identify fibres," Textile Research Journal, vol. 64, no. 8, pp. 444-448, 1994.

[8] M. Sohn, D. S. Himmelsbach, D. E. Akin, and F. E. Barton II, "Fourier transform near-infrared spectroscopy for determining linen content in linen/cotton blend products," Textile Research Journal, vol. 75, no. 8, pp. 583-590, 2005.

[9] C. Ruckebusch, F. Orhan, A. Durand, T. Boubellouta, and J. P. Huvenne, "Quantitative analysis of cotton-polyester textile blends from near-infrared spectra," Applied Spectroscopy, vol. 60 , no. 5, pp. 539-544, 2006.

[10] A. Engelhardt and A. G. S. Arbon, "The fiber year 2007/2008: manmade fibers countinue pace of outgrowing all other fibers," International Fiber Journal, 2008.

[11] T. Matsuo, "Fibre materials for advanced technical textiles," Textile Progress, vol. 40, no. 2, pp. 87-121, 2008.

[12] I. Baer, R. Gurny, and P. Margot, "NIR analysis of cellulose and lactose-Application to ecstasy tablet analysis," Forensic Science International, vol. 167, no. 2-3, pp. 234-241, 2007.

[13] Q. Chen, J. Zhao, C. H. Fang, and D. Wang, "Feasibility study on identification of green, black and Oolong teas using near-infrared reflectance spectroscopy based on support vector machine (SVM)," Spectrochimica Acta A, vol. 66, no. 3, pp. 568574, 2007.

[14] J. Shlens, A Tutorial on Principal Component Analysis, 2005.

[15] T. Lillhonga and P. Geladi, "Replicate analysis and outlier detection in multivariate NIR calibration, illustrated with biofuel analysis," Analytica Chimica Acta, vol. 544, no. 1-2, pp. 177-183, 2005.

[16] K. Krämer and S. Ebel, "Application of NIR reflectance spectroscopy for the identification of pharmaceutical excipients," Analytica Chimica Acta, vol. 420, no. 2, pp. 155-161, 2000. 

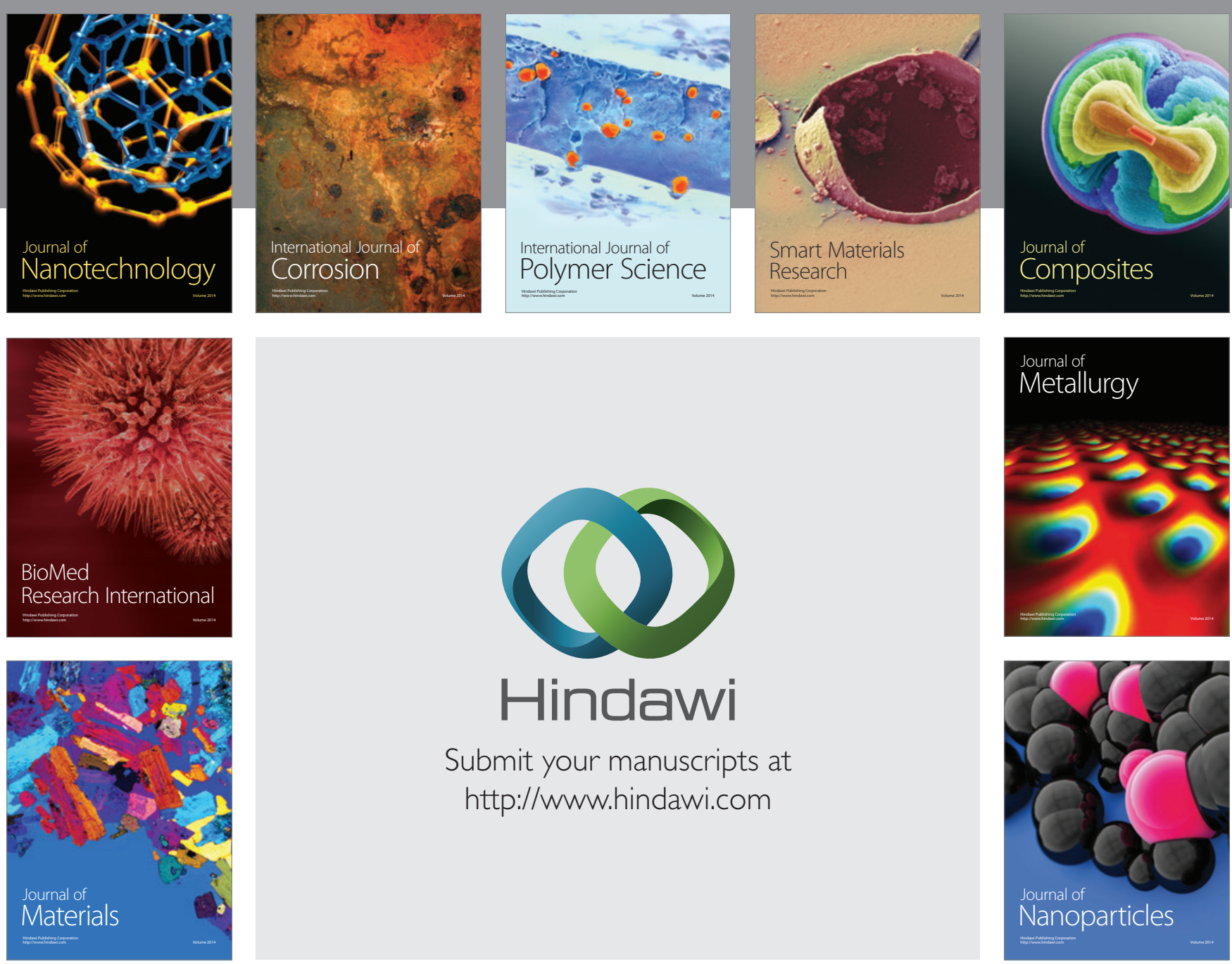

Submit your manuscripts at http://www.hindawi.com
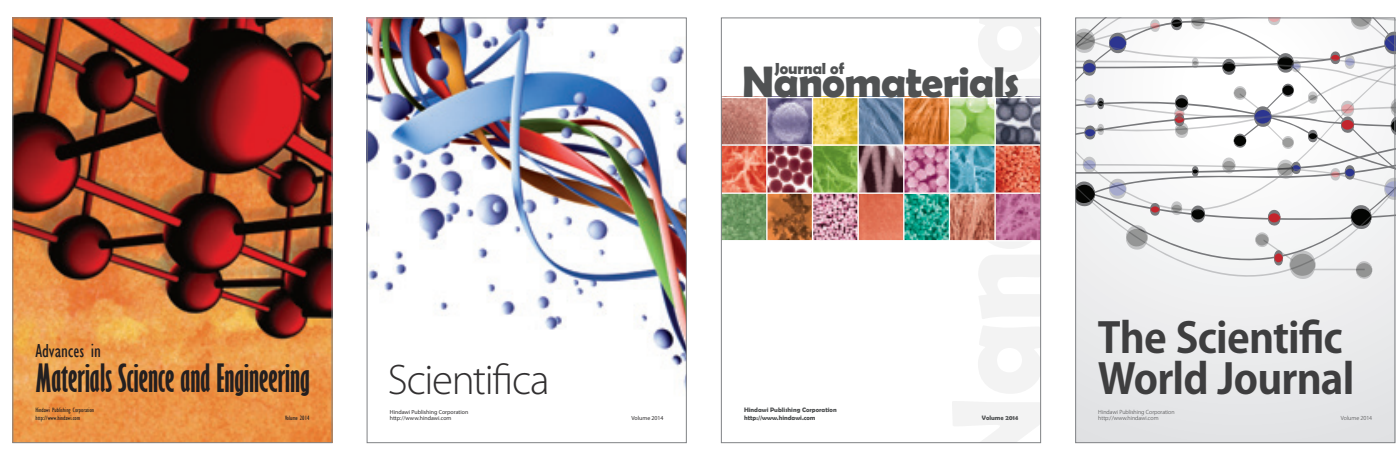

\section{The Scientific World Journal}
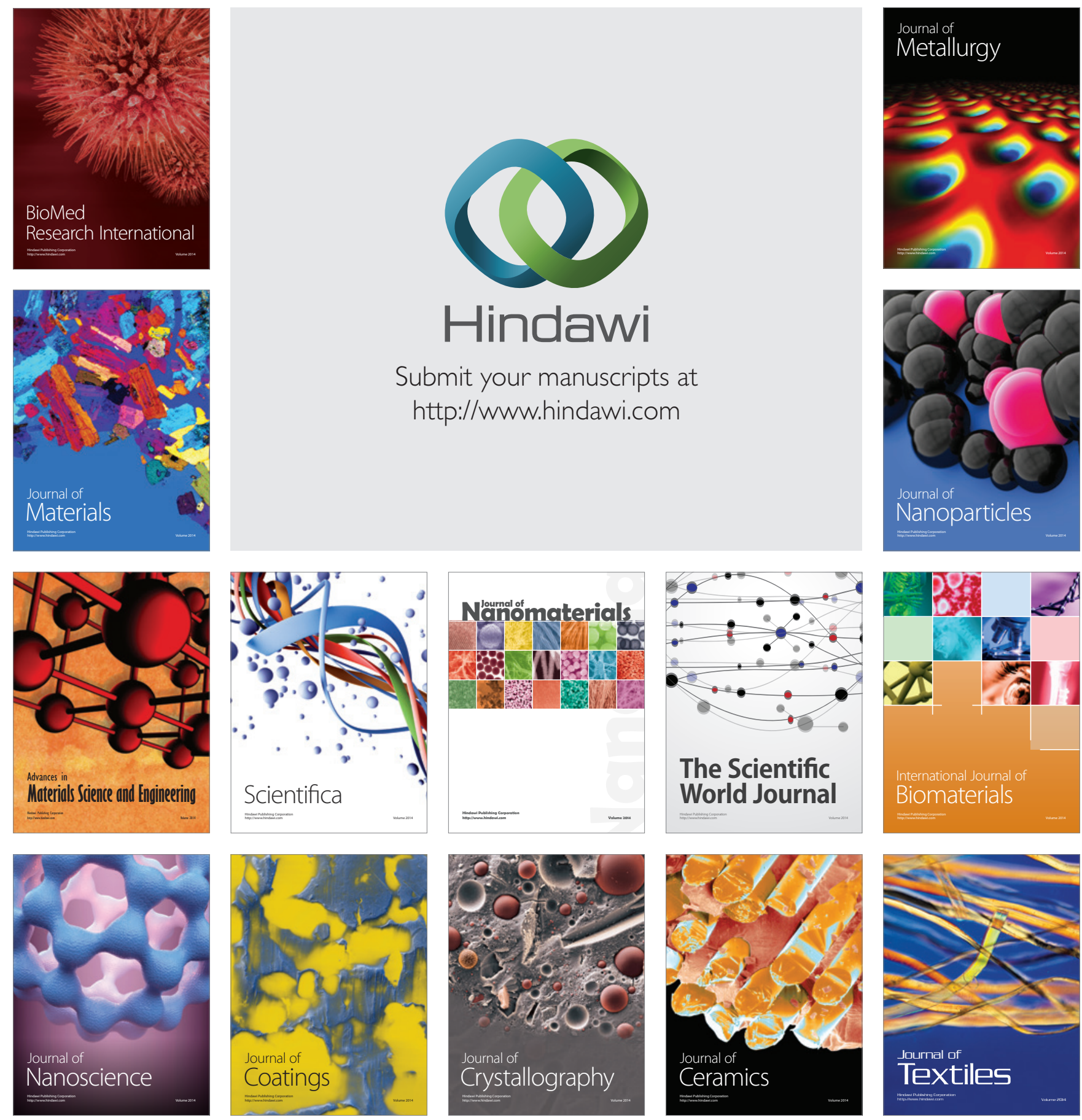